\title{
Functional magnetic resonance imaging of working memory impairment after traumatic brain injury
}

C Christodoulou, J DeLuca, J H Ricker, N K Madigan, B M Bly, G Lange, A J Kalnin, W-C Liu, J Steffener, B J Diamond, A C Ni

Neuropsychology and Neuroscience Laboratory, Kessler Medical Rehabilitation Research and Education

Corporation, 1199 Pleasant Valley Way, West Orange, NJ, 07052, USA

C Christodoulou

J DeLuca

J H Ricker

N K Madigan

B J Diamond

A C Ni

Department of Physical Medicine and Rehabilitation, UMDNJ-New Jersey Medical School, USA C Christodoulou

J DeLuca

J H Ricker

B J Diamond

Department of Radiology

G Lange

A J Kalnin

B M Bly

W-C Liu

Department of

Neuroscience

J DeLuca

Department of

Psychiatry

G Lange

J Steffener

Department of Psychology at Rutgers University, USA

B M Bly

Correspondence to: Dr J DeLuca

delucajo@umdnj.edu

Received 19 June 2000 and in revised form

10 January 200

Accepted 23 January 2001

\begin{abstract}
Objectives-To examine patterns of brain activation while performing a working memory task in persons with moderate to severe traumatic brain injury (TBI) and healthy controls. It is well established that working memory is an area of cognition that is especially vulnerable to disruption after TBI. Although much has been learned about the system of cerebral representation of working memory in healthy people, little is known about how this system is disrupted by TBI.

Methods-Functional magnetic resonance imaging (fMRI) was used to assess brain activation during a working memory task (a modified version of the paced auditory serial addition test) in nine patients with TBI and seven healthy controls.

Results-Patients with TBI were able to perform the task, but made significantly more errors than healthy controls. Cerebral activation in both groups was found in similar regions of the frontal, parietal, and temporal lobes, and resembled patterns of activation found in previous neuroimaging studies of working memory in healthy persons. However, compared with the healthy controls, the TBI group displayed a pattern of cerebral activation that was more regionally dispersed and more lateralised to the right hemisphere. Differences in lateralisation were particularly evident in the frontal lobes.
\end{abstract}

Conclusions-Impairment of working memory in TBI seems to be associated with alterations in functional cerebral activity.

(F Neurol Neurosurg Psychiatry 2001;71:161-168)

Keywords: traumatic brain injury; working memory; functional magnetic resonance imaging

Traumatic brain injury (TBI) in the United States has been estimated to result in over 70000 new cases of disability each year, with a disproportionately large number of teenagers and young adults. ${ }^{1}$ It is well known that cognitive impairment is a major predictor for disability among survivors of $\mathrm{TBI}^{2{ }^{2}}$ and a source of frustration and concern for patients and their families. It is also known that working memory is particularly vulnerable to disruption after TBI. ${ }^{4-6}$ Working memory has been defined as the maintenance of information in a limited capacity temporary storage while performing cognitive manipulations on the information (for example, remembering the address of where you are going while deciding which route to take). ${ }^{7}$ It is important for a wide variety of cognitive skills, such as problem solving, planning, and active listening, and plays a key part in many everyday activities that are essential for return to work (for example, engaging in a telephone conversation while taking notes) and return to school (for example, mental arithmetic). As such, compromised working memory can have significant effects on everyday life.

Working memory normally relies on processing in brain regions that are often selectively damaged by TBI. One key region subserving working memory is the prefrontal cortex. ${ }^{8}{ }^{9} \mathrm{It}$ is well established that in persons with severe TBI, the frontal cortices tend to be damaged, both structurally ${ }^{11}{ }^{11}$ and functionally. ${ }^{12}{ }^{13}$ For instance, PET scanning studies of persons with severe TBI at rest have documented that cerebral hypometabolism, which typically involves the prefrontal cortex, is a frequent outcome. ${ }^{13}{ }^{14}$ It must be noted, however, that physiological and neurobehavioural effects may be widespread beyond the anatomical region suggested by a focal contusion. ${ }^{13} 14$

Much has been learned about the cerebral organisation of working memory in healthy people, most recently through the use of functional neuroimaging techniques. Among the most common findings across various studies involving both verbal and visuospatial stimuli, is activation of the prefrontal and premotor regions of the frontal lobes (for example, involving the middle frontal gyrus or inferior frontal gyrus). ${ }^{89}{ }^{15}$ Parietal activation is also often reported in such studies. ${ }^{9}{ }^{15} 16$ Temporal activation has also been identified, although less commonly. ${ }^{16-18}$

Only one neuroimaging study of working memory in TBI has been published to our knowledge, a study of persons with mild TBI studied within 1 month of injury. ${ }^{19}$ Behaviourally, these patients with TBI did not perform more poorly on the task than the healthy controls. However, relative to controls, the patients with TBI did show increased cerebral activation during tasks that required higher cognitive load, as opposed to tasks with a lesser load, particularly in the right dorsolateral frontal cortex and right parietal lobe. The patients with TBI also demonstrated more widespread (dispersed) regions of activation. Although compelling, it is unclear whether the findings 
obtained in this mild TBI sample would generalise to more severely injured patients. In addition, it is unclear whether the altered pattern of activation found by McAllister et $a l^{19}$ in patients 1 month after injury would continue to be evident in a more chronic TBI sample.

The purpose of this exploratory study was to examine whether decreased working memory performance in patients with moderate to severe TBI is associated with alterations in functional cerebral activity relative to healthy controls. Because of the exploratory nature of this study, it focused on two broadly recognised patterns of cerebral reorganisation in the patients with TBI, patterns that have been found in earlier studies of neurological populations $^{20}$ : specifically, we hypothesised that patients with TBI would show:

(1) Recruitment of remote regions in the contralateral hemisphere, resulting in an alteration in the lateralisation of cerebral activation.

(2) locally expanded recruitment of areas adjacent to those that are active in healthy persons leading to more dispersed cerebral representation.

It has been proposed that these two hypotheses represent the main forms of reorganisation that occur during recovery from brain injury. ${ }^{20}$ Evidence for both these forms of reorganisation have been found in various clinical populations, including stroke, ${ }^{21}$ Alzheimer's disease, ${ }^{22}$ and mild TBI. ${ }^{19}$

\section{Methods \\ SUBJECTS}

Subjects consisted of nine patients who had sustained a moderate or severe TBI and seven healthy controls. The level of injury in the TBI sample ranged from moderate to severe based on scores on the Glasgow coma scale (GCS), ${ }^{23}$ when available, or on other confirmatory data (for example, positive anatomical neuroimaging findings, focal neurological signs, loss of consciousness of 30 minutes or more). The mean GCS score for the patient group was 5.71 (SD 2.14; data unavailable for three patients), and the mean time since injury was 51.33 (SD 41.07) months. Structural MR images of the subjects were taken at the time of testing and examined by a board certified neuroradiologist who was blind to group membership. Three of the patients with TBI displayed clear positive findings. One displayed encephalomalacia in the left posterior temporal lobe. Another showed encephalomalacia in the left temporal, left parietal, left medial frontal, and bilateral inferior frontal regions. The third displayed dark T2 signal consistent with haemosiderin in the right dorsal frontal region.

Demographic data for the two groups are presented in table 1 . All were right handed with the exception of one control subject who was ambidextrous. All subjects gave informed consent, as approved by the institutional review boards of both UMDNJ-New Jersey Medical School and Kessler Medical Rehabilitation Research and Education Corporation (KMRREC). Exclusion criteria consisted of age older
Table 1 Demographic characteristics of the subject groups

\begin{tabular}{llll}
\hline Group & $\begin{array}{l}\text { Mean }(S D) \\
\text { Age }\end{array}$ & $\begin{array}{l}\text { Mean }(S D) \\
\text { Education }\end{array}$ & Male (\%) \\
\hline TBI & $32.67(10.86)$ & $13.89(1.69)$ & 56 \\
Control & $29.71(7.04)$ & $16.17(1.83)^{\star}$ & 57 \\
\hline
\end{tabular}

${ }^{\star} \mathrm{p}<0.05$. TBI $=$ Traumatic brain injury.

than 56 years, previous psychiatric or neurological history (other than TBI for the brain injury group), and pregnancy.

BEHAVIOURAL TASKS

The working memory task consisted of a modified version of the paced auditory serial addition task (PASAT), ${ }^{24}$ a challenging task with significant working memory demands. During the modified PASAT (mPASAT), subjects heard a sequence of numbers, ranging from one to nine, at a rate of one number every 2 seconds. Subjects were instructed to add the first number to the second, the second to the third, and so on, so that they were always calculating the sum of the last two numbers that had been presented. Instead of answering aloud, subjects were told to silently add the numbers, and to lift their right index finger whenever the sum equalled $10 .^{25}$ This modification to the standard PASAT procedure was designed to limit head movement artifacts during image acquisition. Response accuracy was recorded by an observer during image acquisition. In addition to the working memory task, a control task was used in which subjects were asked to imagine that they were brushing their teeth. This control task was designed to demand some degree of attention on the part of the subject, without requiring a significant working memory load. The working memory and control tasks were administered in a fixed sequence that consisted of four sets of alternating 32 second blocks (the experimental task came first in each set). Before the administration of the two tasks, there was one 32 second baseline period. Subjects received task instructions and engaged in practice before entering the magnet.

FUNCTIONAL IMAGING PROCEDURE

All neuroimaging was performed on a General Electric Signa Horizon Echo-speed (1.5 Tesla) MR scanner. Before functional imaging, sagittal T1 weighted localiser images were obtained, followed by whole brain axial $\mathrm{T} 1$ weighted conventional spin echo images for anatomical overlays $(\mathrm{TR}=450, \mathrm{TE}=14$, contiguous $5 \mathrm{~mm}$, $256 \times 256$ matrix, FOV $=24, \mathrm{NEX}=1$ ), yielding an in plane resolution of $0.94 \mathrm{~mm}^{2}$.

Functional imaging consisted of multislice gradient echo images that were acquired with echoplanar imaging (EPI) methods (TE (echo time $)=60 \mathrm{~ms}$; TR (repetition time) $=4000 \mathrm{~ms}$; FOV (field of view) $=24 \mathrm{~cm}$; flip angle $=90^{\circ}$; slice thickness $=5 \mathrm{~mm}$ contiguous). This yielded a $64 \times 64$ matrix with an in plane resolution of $3.75 \mathrm{~mm}^{2}$. A total of 28 images in the axial plane were acquired, providing coverage of the entire brain. A set of coplanar T2 weighted EPI images with identical parameters was also obtained without a task paradigm to 
provide an additional set of T2 weighted structural images.

Subjects performed the working memory and control tasks while lying supine in the scanner. Foam cushioning and tape were used to immobilise the head within the coil to minimise motion degradation. Auditory stimuli were presented to subjects through MRI compatible headphones designed in our laboratory. Sound volume was adjusted so that each participant could adequately hear the stimuli.

ANALYZING IMAGES

Functional MRI data were initially analyzed on a voxel by voxel basis with a general linear model approach, using statistical parametric mapping (SPM96) software. All raw scan data underwent spatial realignment using the SPM96 six parameter model to remove minor (subvoxel) motion related signal change. All scans were spatially normalised to approximate the neuroanatomical atlas of Talairach and Tournoux $^{26}$ using a 12 parameter affine approach and a $\mathrm{T} 2^{\star}$ weighted template image. Scans were then spatially smoothed to $8 \times 8 \times 10$ $\mathrm{mm}$. The SPM maps were thresholded to a stringent $\alpha$ level of 0.001 for assessing specific search regions such as premotor and prefrontal cortex $^{8915}$ as well as lateral parietal ${ }^{915} 16$ and lateral temporal regions ${ }^{16-18}$ identified in previous working memory studies. The threshold method found in SPM96 simultaneously accounts for peak amplitude and spatial extent of clusters to control for type 1 error across multiple comparisons. Spatial extent refers to cluster size $(k)$, the number of adjacent activated voxels that exceed the specified threshold. Talairach labels of activated clusters entailed the use of the Talairach Daemon, a high speed database server for querying and retrieving data about human brain structure over the internet. ${ }^{27}$

\section{Data analysis}

The random effects procedure developed by Holmes and Friston ${ }^{28}$ was used to identify neuroanatomical regions of significant activation in each group. This procedure was also used to identify regions that were significantly more activated in one group versus the other. The random effects procedure eliminates highly discrepant variances between and within subjects in constructing an appropriate error term for hypothesis testing and generalisation to the population. The random effects procedure assumes one scan per subject per condition. Each subject provided two mean images, one from the mPASAT condition and another that combined control and baseline activation. The mean images were offset by two TR (8 seconds) to account for the delay in haemodynamic response.

Additional analyses were performed to further evaluate the two broad general forms of cerebral reorganisation discussed in the introduction:

(1) altered lateralisation-remote activation of the contralateral hemisphere signifying an alteration in the lateralisation of activation.
(2) increased dispersion-locally expanded recruitment of areas adjacent to those that are active in healthy persons (more dispersed cerebral representation). ${ }^{20} \mathrm{~A}$ standard "lateralisation index" was constructed to test for the presence of altered lateralisation in the TBI group, involving greater right lateralised activation on a normally left lateralising type of verbal working memory task. A separate laterality index score was calculated for each subject. The laterality index consisted of the number of activated voxels in clusters in the left cerebral hemisphere minus those in the right hemisphere, divided by the total number of activated voxels within clusters, and then multiplied by $100 \quad(($ left- right $) / \quad($ total $) \times 100)$. Scores ranged from -94 to 100 . Positive scores on the laterality index indicate greater left hemispheric activation whereas negative scores reflect greater right hemispheric activation. It is important to note that the calculation of each subject's laterality index is dependent on that person's particular level of total cerebral activation. Therefore, the use of the laterality index has the benefit of controlling for individual differences in overall activation that are generally present in studies of neuroimaging.

The presence of increased dispersion of activation in the TBI group was tested by use of a "dispersion index," similar to the laterality index described above. This dispersion index indicates the relative proportion of activation in each subject that extended beyond the normally activated areas. The cerebral regions that were most consistently activated by healthy controls in this study were considered to be the normally activated areas (middle frontal gyrus and middle temporal gyrus), in the sense that such activation seemed necessary for healthy persons under the present task demands. Specifically, the dispersion index equalled the number of activated voxels in clusters that were outside the consistently activated regions (representing "diffuse" activation that was dispersed beyond the areas activated by healthy controls) minus those that were within the consistently activated regions, divided by the total number of activated voxels, and then multiplied by 100 . Scores on the dispersion index ranged from -100 to 100 . Using this index, positive scores indicate that most of the activation in that subject was dispersed. By contrast, negative scores indicate that most of the activation was not dispersed.

Group differences for the laterality and dispersion indices were tested by one tailed $t$ tests comparing the two groups on each index. Levene's test for the equality of variances was used to determine whether the $t$ tests to be calculated should assume equal variances in the two groups. In addition to tests of significance, effect sizes (d) were calculated to quantify the magnitude of group differences.

\section{Results}

\section{BEHAVIOURAL DATA}

Patients with TBI made significantly more errors on the mPASAT (mean 6.67 (SD 6.26)) than did controls (mean 1.43 (SD 2.57) 
Table 2 Summary of activated regions during performance on the MPASAT task resulting from random effects analyses thresholded to an a value of 0.001

(Talairach coordinates, cluster size, magnitude)

\begin{tabular}{llll}
\hline & & & \\
Region & $x, y, z(m m)$ & $\begin{array}{l}\text { Cluster } \\
\text { size }\end{array}$ & $Z$ Score \\
\hline (A) control: & & & \\
Frontal lobe & & & \\
Left MFG & $-44,4,42$ & 64 & 5.14 \\
Left MFG & $-22,-2,56$ & 20 & 3.86 \\
Left MFG & $-46,32,24$ & 9 & 3.50 \\
Left MFG & $-38,44,34$ & 1 & 3.15 \\
Left MeFG & $-16,10,48$ & 4 & 3.45 \\
Temporal lobe & & & \\
Left MTG & $-48,-20,-6$ & 93 & 4.85 \\
Left STG & $-64,-44,12$ & 85 & 4.10 \\
Right STG & $52,-36,12$ & 37 & 4.53 \\
Right STG & $58,-54,28$ & 1 & 3.81 \\
Right STG & $56,-20,0$ & 1 & 3.14 \\
Left TTG & $-34,-32,12$ & 1 & 3.10 \\
Parietal lobe & & & \\
Left IPL & $-40,-46,38$ & 2 & 3.13 \\
Right IPL & $56,-38,28$ & 1 & 3.70 \\
Right IPL & $44,-28,22$ & 1 & 3.38 \\
Right IPL & $48,-32,30$ & 3 & 3.32 \\
Right SmG & $36,-48,34$ & 2 & 3.12 \\
Other regions & & & \\
Left sublobar & $-50,-22,16$ & 4 & 3.34 \\
Right sublobar & $30,-26,8$ & 1 & 3.43 \\
Right sublobar & $16,-2,14$ & 2 & 3.12 \\
Left thalamus & $-8,-12,12$ & 22 & 3.38 \\
Right thalamus & $4,-4,2$ & 20 & 3.75 \\
Right thalamus & $18,-10,10$ & 9 & 3.56 \\
Right brainstem & $12,-20,-6$ & 3 & 3.26 \\
Right cerebellum PL & $16,-72,-38$ & 4 & 3.19 \\
$\quad$ & & &
\end{tabular}

(B) TBI:

Frontal lobe

Left IFG

Left PCG

Right MFG

Right MFG

Right MFG

Left SFG

Right MeFG

Temporal lobe

Left MTG

Right MTG

Left STG

Left STG

Left STG

Right STG

Right SubGyra

Parietal lobe

Right SMG

Other regions

Right pons

Left sublobar

$-48,8,2$

$-18,-22,60$

$44,0,52$

$54,34,20 \quad 3$

$38,34,20 \quad 20$

$-2,6$,

$4,0,60$

$-64,-34,0 \quad 14$

$66,-36,-2 \quad 423$

$-64,-44,10 \quad 8$

$-48,-26,4 \quad 24$

$-54,-30,12 \quad 9$

$66,-44,18 \quad 35$

$42,-28,-8 \quad 39$

$46,-46,26 \quad 6$

$2,-24,-22 \quad 4$

Right cingulate gyrus

$-32,-38,20$

$10,20,42$

(C) TBI minus control:

Frontal lobe

Right SFG

Right MFG

Right Subgyral

Parietal lobe

Right PSCG

Occipital lobe

Left cuneus

Other regions

Left cerebellum AL

$8,2,62$

$40,38,-8$

$10,34,0$

16
1
1

$16 \quad 3.68$

$\begin{array}{ll}1 & 3.17\end{array}$

$64,-18,36 \quad 3$

$\begin{array}{lll}-12,-78,6 \quad 6 & 3.40\end{array}$

$-4,-46,-14 \quad 10$

3.36

(D) Control minus TBI:

Frontal lobe

Left SFG

Left MFG

Temporal lobe

Left STG

Right STG

Left FG

Parietal lobe

Right IPL

Right IPL

Occipital lobe

Right LG

Left cuneus

Other regions

Left sublobar

Left thalamus MDN

$\begin{array}{lll}-16,60,26 & 2 & 3.34 \\ -46,36,32 & 1 & 3.19 \\ -42,8,-12 & 11 & 3.52 \\ 60,-18,8 & 2 & 3.17 \\ -52,-44,-16 & 3 & 3.21 \\ & & \\ 64,-34,24 & 7 & 3.51 \\ 44,-28,26 & 8 & 3.48 \\ & & \\ 34,-70,-8 & 39 & 3.49 \\ -14,-74,22 & 2 & 3.20 \\ -20,-12,8 & 25 & 3.62 \\ -6,-10,10 & 5 & 3.31\end{array}$

$(t=2.274, \mathrm{p}=0.044))$. This difference in errors between the two groups amounted to a large effect size $(d=0.79)$. Despite their difficulty with the task, the overall accuracy of the patients with TBI (72.21\% v 94.05\% for controls) indicated that they were able to engage working memory processes in response to the task demands. The errors of both the healthy and TBI groups were almost exclusively omissions. Two of the nine patients with TBI each made a single commission error, whereas the controls did not exhibit any commission errors.

RANDOM EFFECTS ANALYSES

Significant activation in healthy controls was principally located in the left frontal and left temporal lobes, with additional bilateral parietal activation also evident (fig 1, table $2 \mathrm{~A}$ ). Frontal lobe activation occurred predominantly in the middle frontal gyrus. Temporal lobe activation was localised primarily in the middle temporal gyrus) and superior temporal gyrus. Parietal activation occurred primarily in the inferior parietal lobule. Activation among patients with TBI was similar to that of controls, with major foci again displayed in the frontal (for example, middle frontal gyrus) and temporal (for example, middle temporal gyrus, superior temporal gyrus) lobes (fig 1 , table 2 B).

\section{RANDOM EFFECTS ANALYSIS OF ALTERED}

LATERALISATION

Activation was more right lateralised in both the frontal and temporal lobes of the TBI group, whereas the healthy group showed more left lateralised cerebral activation (fig 1; table 2 C and D). Specifically, the TBI group displayed greater activation in regions of the right frontal lobe (superior frontal gyrus, middle frontal gyrus), whereas healthy controls displayed greater left hemispheric activation in these same regions. In the temporal lobe, controls displayed greater activation, predominately in the left hemisphere, especially in superior temporal gyrus. No clear pattern of lateralisation emerged from the activation patterns in the other lobes.

\section{RANDOM EFFECTS ANALYSIS OF INCREASED}

DISPERSION

Activation foci in the frontal lobes of the healthy control group were primarily limited to the left middle frontal gyrus (fig 1, table $2 \mathrm{~A}$ ). In the TBI group, left frontal activation was not found in the middle frontal gyrus, but activation was recruited from the inferior frontal

${ }^{\star}$ Section A of the table lists regions of activation that represent the simple effects of the working memory task for the healthy control group; section B presents those simple effects for the TBI group; section $\mathrm{C}$ presents those areas that were significantly more activated in the TBI group than in the healthy controls; section $\mathrm{D}$ presents those areas that were significantly more activated in the controls than in the TBI group.

$\mathrm{MFG}=$ Middle frontal gyrus; $\mathrm{MeFG}=$ medial frontal gyrus; $\mathrm{IFG}=$ inferior frontal gyrus; $T \mathrm{TG}=$ transverse temporal gyrus; PCG=precentral gyrus; IPL=inferior parietal lobule; $\mathrm{SPL}=$ superior parietal lobule; VLN=ventral lateral nucleus; $\mathrm{SMG}=$ supramarginal gyrus; $\mathrm{WhM}=$ white matter; $\mathrm{AL}=$ anterior lobe of cerebellum; PL=posterior lobe of cerebellum; PSCG=postcentral gyrus; $L G=$ lingual gyrus; $F G=$ fusiform gyrus; $\mathrm{MDN}=$ medial dorsal nucleus. 

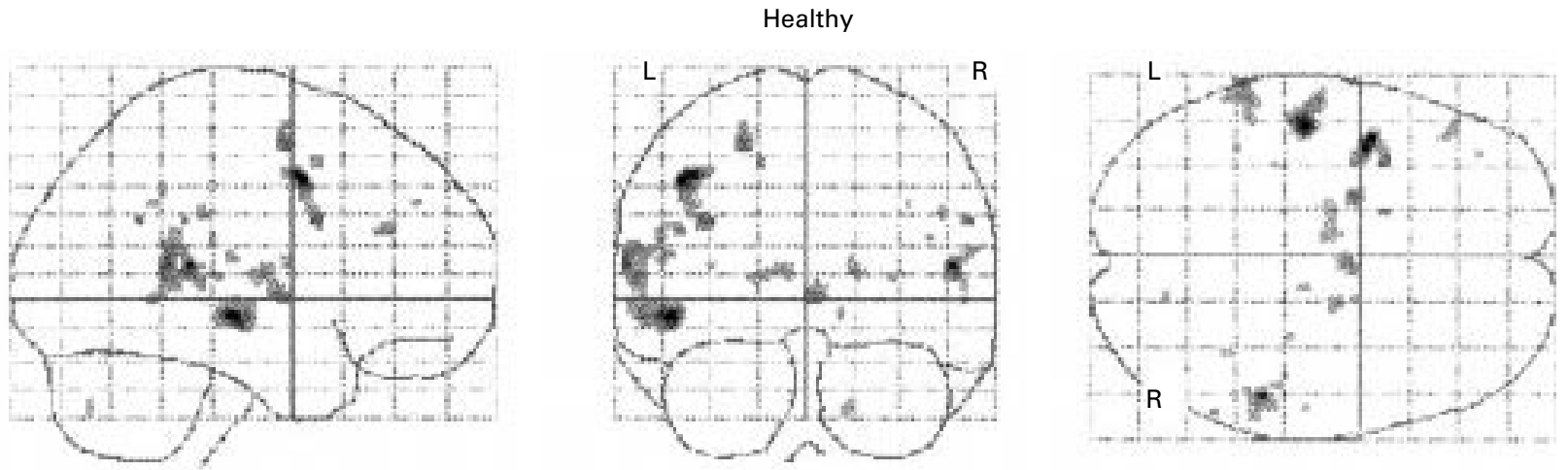

TBI
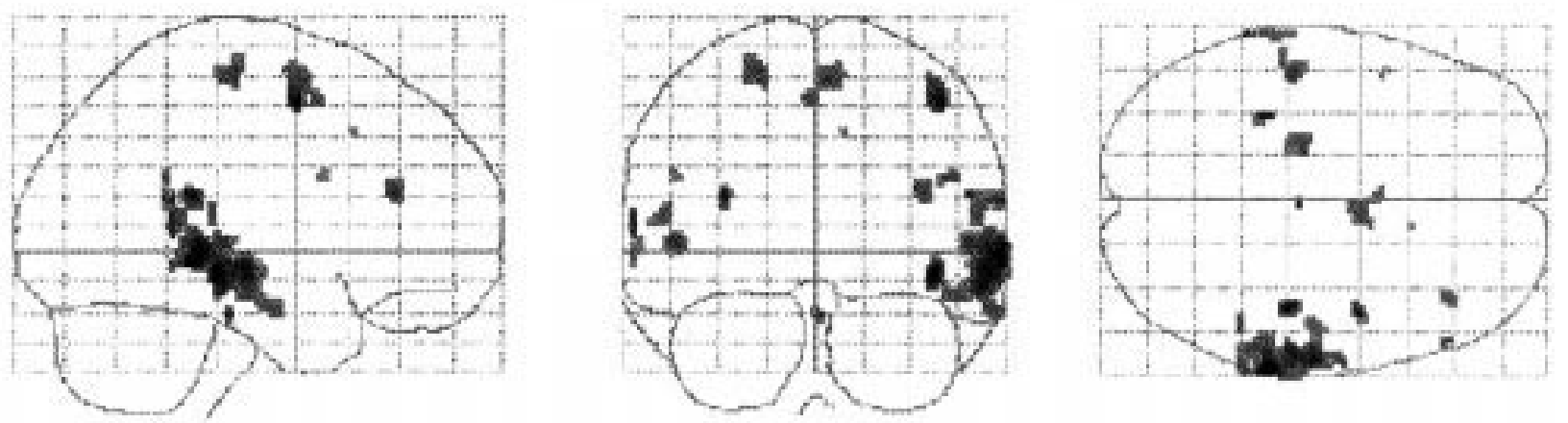

Figure 1 Group activation patterns on the working memory task for the TBI $(n=9)$ and healthy control $(n=7)$ groups. Maximum intensity projections in the three orthogonal views of the brain (sagittal, coronal, and axial) depict areas of significant activation.

gyrus, superior frontal gyrus, and precentral gyrus (fig 1, table $2 \mathrm{~A}$ ). However, in direct comparison of the activation in the two groups (table $2 \mathrm{C}$ and $\mathrm{D}$ ), the above distinctions did not reach significance. In the temporal lobes, both groups tended to display activation primarily in the middle temporal gyrus and superior temporal gyrus. There was no systematic pattern of increased dispersion of activation in other lobes.

LATERALITY INDEX

In addition to the random effects analyses above, group differences in laterality of activation were also assessed by means of the laterality index (see definition above). The TBI group was found to display activation that was significantly more lateralised to the right hemisphere than in the healthy controls across all four lobes combined $(t=-2.041, \mathrm{p}=0.031)$. The TBI group had a negative mean $(-17.53$ (SD 45.60)), indicating greater right hemispheric activation in this group, whereas the healthy controls displayed a positive mean (41.78 (SD 70.61)), indicative of greater left hemispheric activation in these persons. The difference in means between the groups represented a large effect size $(d=0.96)$. Stated another way, five of the six with right lateralised activation (negative laterality index scores) were from the TBI group, whereas five of the seven with left lateralised activation were healthy controls.

LATERALITY INDEX FOR EACH LOBE

To determine whether the overall cerebral laterality effect was specific to particular regions of the brain, laterality indices were created to examine activation in each cerebral lobe separately (using the same basic formula: $\mathrm{L}-\mathrm{R} /$ $\mathrm{L}+\mathrm{R} \times 100$ ). As seen in figure 2 , each lobe tended to display the same trend, though only the laterality index for the frontal lobe reached significance $(t=-1.866, \mathrm{p}=0.042)$. None the less, the effect sizes for all of the differences were moderate (temporal lobe $d=0.53$, parietal lobe $d=0.55$, occipital lobe $\mathrm{d}=0.51$ ) to large (frontal lobe $d=0.88$ ).

DISPERSION INDEX

In constructing the dispersion index (see definition above), two neuroanatomical areasmiddle frontal gyrus and middle temporal gyrus-were identified as representing "focal" activation because they were found to be consistently activated in the healthy controls. The middle frontal gyrus was activated in all six of the controls with significant activation during scanning (one control failed to show activation at the stringent, conservatively chosen threshold of 0.001), whereas five of the six controls displayed significant activation in the middle temporal gyrus. No other areas were as consistently activated in the healthy control subjects. For the purposes of computing the dispersion index, activation outside the middle frontal gyrus and middle temporal gyrus was considered dispersed.

Based on the dispersion index, patients with TBI were found to display significantly more dispersed activation than the healthy controls $(t=1.969, \mathrm{p}=0.035)$. The TBI group displayed a positive mean (46.37 (SD 45.09)), indicating 


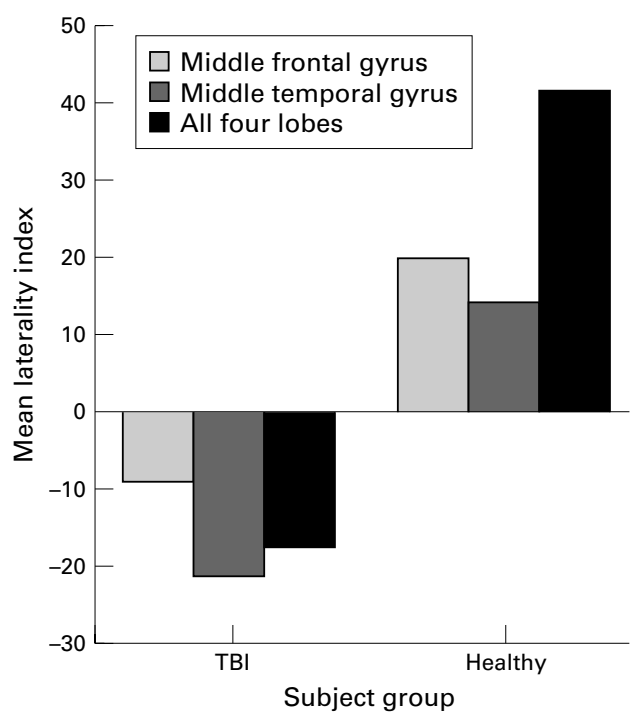

Figure 3 Mean laterality index scores for each subject group in the middle frontal gyrus and middle temporal gyrus during performance of the MPASAT (positive scores indicate relatively more left hemispheric activation, negative scores represent relatively more right sided activation).

relatively more dispersed activation in this group, whereas the healthy controls displayed a negative mean (-10.69 (SD 70.74)) indicative of relatively more focal activation in these subjects. This difference represented a large effect size $(d=1.00)$. Stated another way, six of the nine with more dispersed activation (positive dispersion index scores) were from the TBI group, whereas three of the four with less dispersed activation (negative dispersion index scores) were healthy controls.

LATERALITY INDEX FOR ACTIVATION IN AREAS OF FOCAL ACTIVATION

To determine whether lateralisation was particularly evident in the areas of consistent activation in healthy controls, separate laterality indices were created for the middle frontal gyrus and middle temporal gyrus. As seen in

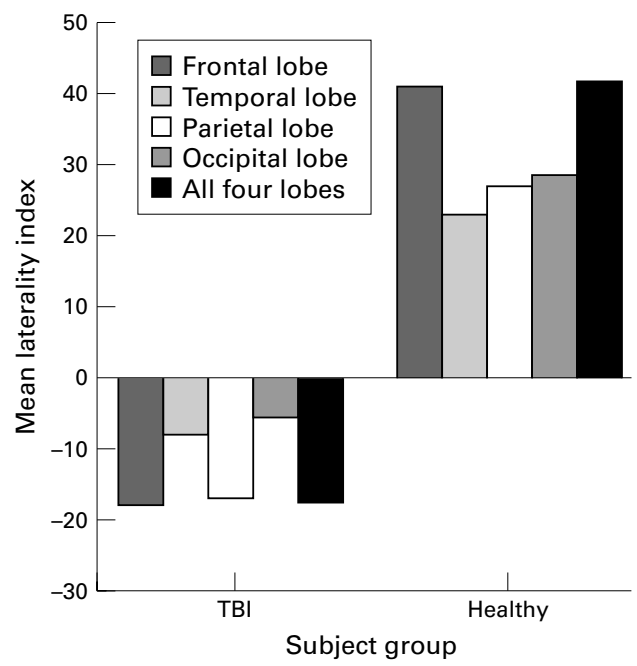

Figure 2 Mean laterality index scores for each subject group separated by lobe, indicating the degree to which cerebral activation was lateralised during performance of the MPASAT (positive scores indicate relatively more left hemispheric activation, negative scores represent relatively more right sided activation). figure 3, these two areas displayed the same general lateralisation trend as found above for the larger regions (activation in the TBI group was more right lateralised than in healthy controls), though the differences did not reach significance. The effect sizes of these differences were small (for middle frontal gyrus, $d=0.35$ ) to medium (for middle temporal gyrus, $d=0.52)$.

\section{Discussion}

The present study examined two general patterns of cerebral reorganisation after moderate and severe TBI, based on findings in other clinical populations ${ }^{20}$ :

(1) recruitment of remote regions in the contralateral hemisphere resulting in an alteration in the lateralisation of activation).

(2) Locally expanded recruitment of areas adjacent to those that are active in healthy persons resulting in more dispersed cerebral representation.

The present study provides the first published evidence to our knowledge that persons with moderate and severe TBI show altered cerebral activation during the processing of working memory tasks, relative to healthy controls. The fact that cerebral activation was present in the patients with TBI, despite their difficulty in performing the task, suggests that fMRI can play an important part in characterising the neurofunctional correlates of cognitive impairment in this patient population.

The results of the present study show that, in general, the same distributed network was activated in the brain during a working memory task in patients with TBI as that found in the healthy control group. Both the TBI and healthy control groups showed cerebral activation in regions of the frontal, temporal, and parietal lobes of the brain. Previous neuroimaging studies of working memory performance in healthy subjects have identified similar regions as crucial components in this distributed network. In particular, middle frontal gyrus activation displayed by both groups in the present study is one of the most common findings in previous verbal working memory studies of healthy persons, ${ }^{8929}$ including a working memory study involving arithmetical calculations. $^{30}$ Patients with mild TBI who were examined within 1 month of injury have also been found to display activation of the middle frontal gyrus during working memory. ${ }^{19}$ Parietal activation in areas such as the inferior parietal lobule ${ }^{9}$ and supramarginal gyrus ${ }^{9}{ }^{16}$ has also been commonly reported in the literature. Little parietal activation was seen in the present study, but this may be related to the tasks selected. Temporal lobe activation, in areas such as the superior temporal gyrus, is less common in working memory paradigms with healthy subjects. ${ }^{16-18}$ Some researchers have interpreted such temporal activation as reflecting part of the articulatory loop of verbal working memory. ${ }^{16}{ }^{17}$ The relatively large clusters of temporal activation in middle temporal gyrus and superior temporal gyrus displayed by both groups in the present study may, in part, be 
associated with the phonological processing of auditory stimuli in the working memory task.

Despite general similarities between groups in regional brain activity, important differences were also found between the TBI and healthy groups. The cerebral activation in the TBI group was lateralised more towards the right hemisphere and away from the left in comparison with the healthy controls. This lateralised difference was especially evident within the frontal lobes. The reason for the relative increase in right hemispheric activation in the TBI group is unclear, although it is possible that it resulted from decreased efficiency in the neural regions normally responsible for task processing or that the two groups differed in their approach to processing the task, or both.

The results of previous studies of both healthy patients and patients with mild TBI suggest that the difficulty displayed by the TBI group in performing the task may have played a part in the lateralised difference. Two neuroimaging studies have found that better performance on verbal working memory tasks is associated with increased left hemispheric activation in frontal ${ }^{18}{ }^{19}$ and parietal sites. ${ }^{18}$ By contrast, poorer performance was found to be associated with increased activation in right frontal and parietal sites. ${ }^{18}$ In the present study, group differences in lateralised activation were clearly evident in the frontal lobes, but less obvious within the parietal lobes.

One variable that may impact on performance of working memory tasks is working memory load. The literature generally supports a model of brain activation in which there is an increased haemodynamic response in relation to increasing task difficulty. In the present study, it is possible that performance of the task required more cognitive "effort" for the TBI group than for healthy persons because of damage to the underlying neural substrate that maintains and manipulates information in the working memory system. Some previous studies of healthy subjects and one study of patients with mild TBI have found that increased verbal working memory load is associated with increased right hemispheric activation in regions of the frontal ${ }^{19} 3132$ or parietal lobes. ${ }^{17}{ }^{19}$ Yet some of the studies that have examined the relation between working memory load and activation have found that not all positive correlations were with regions of the right hemisphere. ${ }^{915}{ }^{17}$ However, even the findings of many of these studies suggest that on the whole, right hemispheric clusters encompassed a greater volume ${ }^{917}$ or were more common ${ }^{15}$ than those on the left. Taken together, the results of the present study, in conjunction with data from healthy subjects in previous studies, suggest that altered cerebral activation is a "normal" response to increased working memory load or decreased perfomance, or both

Another neuroimaging finding that may be relevant to the present verbal working memory results is that aphasic patients show increased activation of right hemispheric regions during language testing. ${ }^{33}$ Although the relatively diffuse and multifocal nature of damage after
TBI argues against drawing strong parallels between the two sets of findings, it does seem that the patients with TBI in the present study required the recruitment of the right hemispheric regions in an effort to process a working memory task that is normally left lateralised. It is unclear, however, if such patients would display similar recruitment of the left hemisphere in the performance of a working memory task that is normally right lateralised. Among the possible hypotheses regarding the impact of TBI on the pattern of cerebral activation during working memory are (1) recruitment of the contralateral hemisphere - that is, brain activation becomes lateralised toward the hemisphere not normally activated in healthy subjects by such a task; (2) recruitment of the right hemisphere - that is, brain activation becomes lateralised toward the right hemisphere regardless of the task. Future studies can be designed to help to differentiate between these two potential patterns.

The results of the analysis examining whether patients with TBI would display a more dispersed pattern of cerebral activation were mixed, depending on the analysis conducted. The dispersion index analysis showed that the TBI group did indeed display a more dispersed activation in regions immediately surrounding the area of interest (middle frontal gyrus and middle temporal gyrus). This finding may represent an attempt by the brain to engage additional regional cerebral resources to complete the task, similar to the increased cerebral representation seen using motor tasks. ${ }^{35}$ By contrast, the results of the random effects analysis did not show a significant increase in more dispersed or widespread cerebral activation, although the analysis approached significance. It is likely that with an increased sample size, this analysis would have become significant with the random effects analysis.

The present study is properly regarded as exploratory and interpretation of the findings requires the consideration of several issues. The TBI population as a whole is by no means homogenous, and individual differences among patients (as well as the controls) could certainly account for some of the variability in task performance, brain organisation, and brain reorganisation. Assessment of cognitive functioning was limited to the measurement of working memory task performance while in the scanner. Although the TBI sample was clearly impaired on that task, further characterisation of their cognitive functioning with "out of scanner" measures should be incorporated into future studies. It is possible that the relatively high error rate of the TBI group on the working memory task complicates interpretation of their activation results. However, it should be noted that another study found that patients with mild TBI activated cerebral regions similar to those found in the present study, while performing working memory tasks on which they displayed no behavioural deficits compared with healthy controls. ${ }^{19}$ The vast majority of the errors exhibited by the subjects in the present study were omissions. The presence of 
omissions may suggest difficulty with sustained concentration or maintenance of information. However, the study was not designed to distinguish where the breakdown in information processing occurs. For example, performance of the mPASAT seems to require multiple working memory components, including the supervisory capabilities of the central executive (for example, in allocating working memory resources for the continual updating of the contents of working memory and the mental calculation of arithmetical sums) in addition to the more primary processing activities of the phonological loop (for example, in initially encoding auditory verbal stimuli into working memory). The task was chosen in part because the PASAT is a standard task that has long been used in the assessment of patients with TBI. ${ }^{4}$ Future studies should focus on more precise measurements of where within the working memory system patients with TBI show deficient processing. Certainly, a substantial amount of additional research is necessary before the theoretical details and clinical implications of this work are fully known.

Future research is needed to clarify several other issues raised by the present study. It will be necessary to more clearly examine the issue of lateralisation of activation after TBI. The present results may be a unique function of the paradigm employed (it may have required a disproportionate amount of left hemispheric processing), thus additional paradigms that use tasks known to specifically activate the right hemisphere will be needed. It will also be of interest to examine working memory across the range of initial injury severity (from mild through severe). Further research is needed that manipulates multiple task parameters to tease apart the effects of task difficulty, cognitive load, and cerebral reorganisation. Improved data analysis techniques will allow for greater consideration of possible "false positive" activations in brain regions. Future functional imaging studies of TBI can explore whether changes in neural organisation of function occur during the course of clinical treatment. Finally, the information derived from fMRI studies of how working memory functions are reorganised after TBI may provide greater insight into how working memory is organised in the healthy brain.

This research was supported in part by grants from the Henry $\mathrm{H}$ Kessler Foundation and the Hyde and Watson Foundation. We thank Allison Gray and Chris Pernell for their help with thank Allison Gray and Chris Pernell for their help with scanning and d
tistical advice.

1 Kraus JF, McArthur DL. Incidence and prevalence of, and costs associated with, traumatic brain injury. Rosenthal $M$, costs associated with, traumatic brain injury. Rosenthal M,
Griffith ER, Kreutzer JS, eds. Rehabilitation of the adult and Griffith ER, Kreutzer JS, eds. Rehabilitation of the adult and
chld with traumatic brain injury. 3rd ed. Philadelphia, PA: FA

2 Ruff RM, Marshall LF, Crouch J, et al. Predictors of outcome following severe head trauma: follow-up data from the Traumatic Coma Data Bank. Brain Inj 1993;7: 101-11.

3 Kaplan CP, Corrigan JD. The relationship between cognition and functional independence in adults with traumatic brain injury. Arch Phys Med Rehabil 1994;75:643-7.

4 Levin HS, Gary HEJr, Eisenberg HM, et al. Neurobehavioral outcome 1 year after severe head injury. 7 Neurosurg 1990;73:699-709.
5 McDowell S, Whyte J, D'Esposito M. Working memory mpairments in traumatic brain injury: evidence from dual task paradigm. Neuropsychologia 1997;35:1341-53.

6 Stuss DT, Ely P, Hugenholtz H, et al. Subtle neuropsychological deficits in patients with good recovery after closed head injury. Neurosurgery 1985;17:41-7.

7 Baddeley AD. Working memory. Oxford, UK: Oxford University Press, 1986

8 Cohen JD, Forman SD, Braver TS, et al. Activation of the prefrontal cortex in a nonspatial working memory task with functional MRI. Hum Brain Mapp 1994;1:293-304.

9 Braver TS, Cohen JD, Nystrom LE, et al. A parametric study of prefrontal cortex involvement in human working memory. Neuroimage 1997;5:49-62.

10 Levin HS, Benton AL, Grossman RG. Neurobehavioral consequences of closed head injury. New York: Oxford University Press, 1982.

11 Zafonte RD. Neuroimaging in traumatic brain injury. In: Horn LJ, Zasler ND, eds. Medical rehabilitation of traumatic brain injury. Philadelphia, PA: Mosley, 1996:251-70.

12 Jansen HML, van der Naalt J, van Zomeren AH, et al. Cobalt- 55 positron emission tomography in traumatic brain injury: a pilot study. $\mathcal{f}$ Neurol Neurosurg Psychiatry 1996;60:221-4.

13 Langfitt TW, Obrist WD, Alavi A, et al. Computerized tomography, magnetic resonance imaging, and positron emission tomography in the study of brain trauma: preliminary observations. F Neurosurg 1986;64:760-7.

14 Fontaine A, Azouvi P, Remy P, et al. Functional anatomy of neuropsychological deficits after severe traumatic brain injury. Neurology 1999;53:1963-8.

15 Cohen JD, Perlstein WM, Braver TS, et al. Temporal dynamics of brain activation during a working memory task. Nature 1997;386:604-8

16 Paulesu E, Frith CD, Frackowiak RSJ. The neural correlates of the verbal component of working memory. Nature 1993; 362:342-5.

17 Salmon E, Van der Linden M, Collette F, et al. Regional brain activity during working memory tasks. Brain 1996;119:1617-25.

18 Seidman LJ, Breiter HC, Goodman JM, et al. A functional magnetic resonance imaging study of auditory vigilance with low and high information processing demands. Neuropsychology 1998;12:505-18.

19 McAllister TW, Saykin AJ, Flashman LA, et al. Brain activation during working memory 1 month after mild traumatic brain injury: a functional MRI study. Neurology 1999;53: $1300-8$

20 Chollet F, Weiller C. Imaging recovery of function following brain injury. Curr Opin Neurobiol 1994;4:226-30.

21 Weiller C, Ramsay SC, Wise RJS, et al. Individual patterns of functional reorganization in the human cerebral cortex after capsular infarction. Ann Neurol 1993;33:181-9.

22 Saykin AJ, Flashman LA, Frutiger SA, et al. Neuroanatomic substrates of semantic memory impairment in Alzheimer's disease: patterns of functional MRI activation. 7 Int Neuropsychol Soc 1999;5:377-92.

23 Teasdale G, Jennett B. Assessment of coma and impaired consciousness: a practical scale. Lancet 1974;ii:81-4.

24 Roman DD, Edwall GE, Buchanan RJ, et al. Extended norms for the paced auditory serial addition task. Clin Neuropsychol 1991;5:33-40.

25 Lange G, DeLuca J, Maldijan JA, et al. FMRI assessment of cerebral activation in response to an auditory working memory task in patients with chronic fatigue syndrome. Neuroimage 1998;7:S853.

26 Talairach J, Tournoux P. Co-planar stereotaxic atlas of the human brain. Stuttgart, Germany: Thieme, 1988.

27 Lancaster JL, Summerln JL, Rainey L, et al. The Talairach Daemon, a database server for Talairach atlas labels. Neuroimage 1997;5:5634.

28 Holmes AP, Friston KJ. Generalisability, random effects, and population inference. Neuroimage 1998;7:S754

29 Petrides M, Alivasatos B, Meyer E, et al. Functional activation of the human frontal cortex during the performance of verbal working memory tasks. Proc Natl Acad Sci USA 1993;90:878-82.

30 Burbaud P, Degreze P, Lafon P, et al. Lateralization of prefrontal activation during internal mental calculation: a functional magnetic resonance imaging study. 7 Neurophysiol 1995;74:2194-9.

31 Manoach DS, Schlaug G, Siewart B, et al. Prefrontal cortex fMRI signal changes are correlated with workiing memory load. Neuroreport 1997;8:545-9.

32 Rypma B, D'esposito $M$. The roles of prefrontal brain regions in components of working memory: effects of memory load and individual differences. Proc Natl Acad Sci USA 1999;96:6558-63.

33 Papanicolaou AC, Moore B, Deutsch G, et al. Evidence for right hemisphere involvement in recovery from aphasia. Arch Neurol 1988;45:1025-9.

34 Ohyama M, Senda M, Kitamura S, et al. Role of the nondominant hemisphere and undamaged area during word repetition in poststroke aphasics: A PET activation study. Stroke 1996;27:897-903.

35 Miyai I, Yasuda T, Suzuki T. Longitudinal function magnetic resonance imaging study in hemispheric stroke [abstract]. Ann Neurol 1999;46:467. 